\title{
AUTONOMOUS ULTRASONIC BASED WATER LEVEL DETECTION AND CONTROL SYSTEM
}

\author{
M. I. Bello1, ${ }^{*}$, S. M. Gana ${ }^{2}$, M. I. Faruk ${ }^{3}$ and M. J. Umar ${ }^{4}$ \\ 1, 2, 3, 4 Department of Physics and Electronics, Bayero University, Kano, Kano State. NiGERIA \\ E-mail addresses: ${ }^{1}$ mibello.elt@buk.edu.ng, ${ }^{2}$ sgana@live.com,3fabolouskay@yahoo.com, \\ 4 mainumajafar15@gmail.com
}

\begin{abstract}
This paper presents an automated non-intrusive control system for monitoring the water level of domestic overhead and underground reservoir tank base on the property of wave reflection. The system consists of two HC-SR04 Ultrasonic transceivers that generate ultrasonic pulses and determines the depth of the water surface based on the total Time of Flight (TOF) of the reflected wave. An ATMEGA328 microcontroller was programmed to read the sensors, control the water level and display the corresponding volume of the water on a Liquid Crystal Display (LCD). The experimental result proves the system stability both at turbulence and laminar flows. The proposed approach can be extended to monitor and control the volume and level of other valuable fluids such as diesel, kerosene etc;; as well as hazardous chemical where human interventions may be treacherous.
\end{abstract}

Keywords: Ultrasonic, Transceiver, Time of flight, Liquid Crystal Display.

\section{INTRODUCTION}

Water is the one most essential commodity among all living things. Major sources of water include rain, tapped, river and underground water. It plays a very vital role in agricultural, transportation, fishing, and industrial sectors as well as for household and recreational application. These reasons in conjunction with the high demand and limited supply for it together with the rapidly increasing population make it necessary to reserve water in dams, overhead and underground tanks for future application.

A large amount of water is being wasted especially during the reservation; it is a common practice in homes and industries to be notified that their reservoir tank is filled to brim only when the reservoir begins to overflow which by implication results in a large quantity of water lost which may be useful to another teaming populist.

Several attempts were made to mitigate the water waste using mechanical devices such as the ball gate and electronically using electronics circuitries which are in most cases mostly intrusive in nature. Most of the electronic approaches in literature involve the use of dielectric capacitive method [1] or metal conducting probes that have direct contact with the water in the reservoir at regular graduated intervals as shown in Figure 1, the direct contact with the water leads to the following problems. Firstly, electrical contact with water entailed current passage from one metal contact to the other through the water molecules, passage of current through water decomposed the water into other chemical elements (electrolysis) that will entirely change its property and render it unhealthy for human consumption[2]; secondly, metal that have direct contact with water causes corrosion on the metal probes and thereby decrease the efficiency of the system[3]; thirdly, to achieve a more reasonable resolution and precision, large number of conducting probes need to be used which makes the system to be highly intrusive and invasive. Owing to the aforementioned predicaments among others, it deems necessary to develop a more efficient and robust approach to conserve water effectively.

\section{RELATED LITERATURE}

There are many proposed methods for liquid level detection given in various literature; such as the capacitive, fibre optics, infrared techniques etc. A more recent and related work proposed the level detection using ultrasonic sensor [4], but the system dynamics 
and the input to the system were not taken into consideration which will render the system highly unstable. Ebere, et al [5] used copper sensors positioned at a particular level in the tank as the water level sensor. He considers the electrical conductivity of water and immersed metal contacts (copper) to compare the voltage corresponding to the water level with a reference voltage. In another related research, a water level controller was designed using Microcontroller interfaced with a couple of sensors, buzzer, motors and LCD for the water level detection, alerting, flow control and display respectively proved to have some level of sturdiness in terms of cost, speed and flexibility, though some degree of complexity is involved in relation to the overall system integration. It can be observed that the system require high-level expertise in its development, ranging from the sensor selection to the development of the signal conditioning circuit unit, then data acquisition which is usually accompanied by computer programming and finally to the output display unit for observations[6].

Sakharov et al. proposed a liquid level sensor by using ultrasonic lamb wave where the characteristics of the acoustic lamb wave propagating in a steel plate was observed experimentally, but the non-linearity of the system renders it impracticable[7]. Guillet et al. [8] proposed an innovative way of detecting water level, by using Chipless RFID principles. Several high quality factor resonators are used for this purpose where no additional sensitive material is needed. The water level detection is done using classical RFID tag with Chipless RFID sensor design based on multiple resonators etched on flexible lamination. The maximum water level that can be detected is strongly dependent on the distance between the tag and the reader antenna. N. K. Kaphungkui [9] utilizes the switching effect of a transistor which will be either in cut off mode or saturation mode depending upon the signal level applied to its base and a 555 timer control the state of output. He also proposed checking the level of impurity in the water (dirt) where low Signal is receive from the output. A relay is used to switch the pump ON only when the High output is produce from both dirt detection and when water level is low otherwise pump is off. On the other hand an invasive optical fiber technique is used for the detection of liquids below freezing point; the system was made up of a multiplexed array of point probes though limited to the elimination of large temperature gradient from liquid vapor[10]. In another related literature, a water level controller was developed using a metal contact and the concept of water conductivity to detect its maximum and the minimum levels. The sensing unit must be fully immersed into the water tank before any measurement can be obtained with make the system highly intrusive. Other disadvantages of the designed system is its unreliability due to water-metal contact that will lead to corrosion and the fact that the system only consider the level extremes (maximum and the minimum) and ignore all other levels [11].

\section{METHODS}

This paper presents an efficient, non-intrusive and contactless means of detecting the level of water in a reservoir by using an ultrasonic transceiver sensor. The ultrasonic sensor is a convenient way of measuring distances from an object. The sensor possesses high accuracy within the range of $2 \mathrm{cmto} 500 \mathrm{~cm}$ from the target object with a resolution of $0.3 \mathrm{~cm}$. The sensor transmits ultrasonic pulses of $40 \mathrm{kHz}$ frequency square wave through the transmitter; the echo received due to the target is then picked-up by the receiver module, the receiver then produce a signal wave form with a time period that is proportional to the distance of the object from the sensor. A suitable microcontroller is used to interpret the received signal.

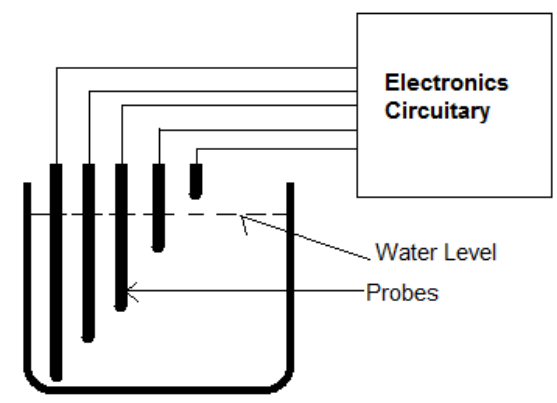

Figure 1: Intrusive Water level Detection System

\subsection{Water Level Sensing}

To detect the level of water in the reservoir, a sensor is required such as the infrared optical sensor [12]. In this work, the HC-SR04 ultrasonic range finder was used, the ultrasonic sensor transmits short, high-frequency sonic pulses at regular intervals, these pulses propagate in air at the velocity of sound $\left(340 \mathrm{~ms}^{-1}\right)$. Like any other wave, the ultrasonic wave exhibits the property of reflection[13].Wave reflection is the change in the direction of a wavefront at an interface between two different media so that the wavefront returns to the medium which is originated from[14]. When the transmitted pulse wave strike an obstacle (in this case the surface of water in a container), the wave undergoes specular reflection and bounce back to the sensor receiver as shown in Figure 2. The level of the water in the container is determined by the distance between the sensor and the level of the liquid by which the reflection occur. As the level of the liquid decreases, 
the distance between the sensor and liquid level increases. The distance is determined by measuring the total travel time and not by the intensity of the sound. To compute the distance between the sensor and the level off the liquid, let $d x$ and $d$ trepresents a certain distance from the sensor and the travel time of the incident and reflected wave respectively. The total time of flight of the ultrasonic burst from the sensor to the level of the liquid and back to the receiver is given by $2 d x$, therefore the speed $S$ of the wave is given by;

$$
S=\frac{2 d x}{d t}
$$

This implies that

$$
d x=\frac{S d t}{2}
$$

Therefore:

$$
\int d x=\frac{S}{2} \int d t
$$

Given the speed of sound in air to be approximately $340 \mathrm{~ms}^{-1}$ or $34000 \mathrm{cms}^{-1}$

The instantaneous distance $x$ is calculated as

$$
x=\frac{34000 \mathrm{cms}^{-1} \times d t}{2}=17000 \mathrm{cms}^{-1} \times t
$$

Therefore, the instantaneous distance of an object $x$ placed away from the sensor with respect to the wave time of flight $t$ can be computed by eq.(4). Furthermore, if $H$ is the maximum height of the tank and $v$ is the volume of the liquid in the tank, it follows that

And

$$
d l=H-d x
$$

$v=k d l$

Where $k$ is constant of proportionality.

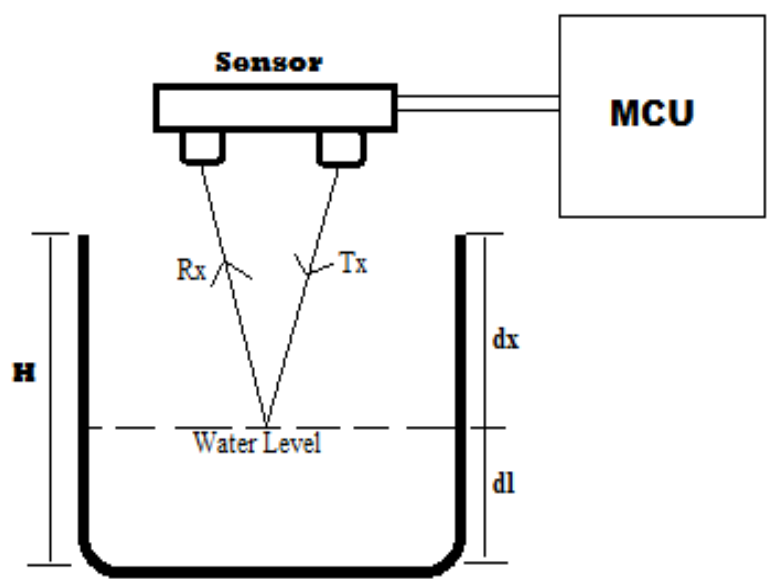

Figure 2 Range Finding Using Ultrasonic Sensor

\subsection{Data processing}

To process the data sent to and received from the Ultrasonic sensor, a microcontroller is required such as Atmel 89C52 used by [6]. In this work, an
ATMEGA328P microcontroller was used. The microcontroller was programmed to concurrently perform the following tasks:

- Sends ultrasonic signal at $40 \mathrm{kHz}$ frequency through two of its digital output pins that are connected to two trigger pins of the ultrasonic sensors, one for the overhead and the other for the underground reservoir.

- To receive the reflected signal from the echo pins of the sensor, process the data and compute the corresponding level of the liquid in each of the reservoirs.

- To actuate the electric pumping machine to pump water from the underground to the overhead tank with the condition that there exists sufficient quantity of water in the underground tank so as to avoid running the pumping machine without water.

- To automatically shut down the pumping machine whenever the level of the water in the overhead tank reaches the maximum capacity of the reservoir tank to avoid over flooding.

- To display the level of water in each tank reservoir and the state of the pumping machine on a16x2 alphanumeric liquid crystal display (LCD).

\subsection{Water Pump Actuator}

This unit is achieved by using a transistor for switching a relay and the relay control an AC contactor for switching a low power PUMP as shown in Figure 3.

A general purpose medium power BC547 (BJT) transistor was used for this project design and it has the following specifications:

$\mathrm{I}_{\mathrm{c}(\max )}=100 \mathrm{~mA}, \mathrm{~h}_{\mathrm{fe}}=110 \sim 220, \mathrm{~V}_{\mathrm{CEO}}=50 \mathrm{~V}$, Frequency $=300 \mathrm{MHz}, \mathrm{V}_{\mathrm{CE}(\mathrm{sat})}=0.6 \mathrm{~V}$, Power dissipation $=0.6 \mathrm{~W}$, $\mathrm{V}_{\text {min }}= \pm 5 \mathrm{~V}, \mathrm{~V}_{\mathrm{BE}}=900 \mathrm{mV}$. The winding resistance of the $D C$ relay used is $R_{w}=100 \Omega$.

Therefore;

$$
\begin{gathered}
I_{C(\text { sat })}=\frac{V_{C C}-V_{C E(\text { sat })}}{R_{w}}=\frac{5-0.6}{100}=0.044 \mathrm{~A} \\
I_{C(\text { sat })}=44 \mathrm{mABut} I_{B}=\frac{I_{C}}{h_{f e}}=\frac{0.044}{120}=0.367 \mathrm{~mA} \\
R_{3}=R_{B}=\frac{V_{\text {min }-V_{E B}}}{I_{B}}=\frac{5-0.9}{0.000367}=11171.66 \Omega \\
\approx 11.17 \mathrm{~K} \Omega \text {. A standard value of } 10 \mathrm{~K} \text { resistor was used for } R_{3}
\end{gathered}
$$




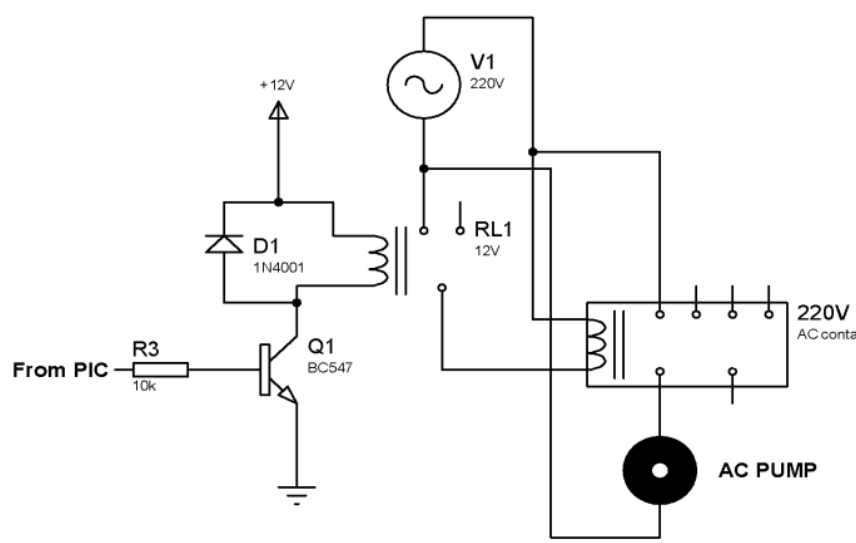

Figure 3: Pump Control Circuit

\section{RESULT AND DISCUSSION}

The contactless autonomous liquid level control system was designed using the ATMEGA328P microcontroller, HC-SR04 ultrasonic range finder, a 30watt AC pump and a Hitachi HD44780 16x2 LCD display. The system is simulated using the Proteus simulation platform.

\subsection{Simulation}

The program was written on the Arduino sketch and the simulation diagram is shown in Figure 4. Two $10 k \Omega$ potentiometers were used to serve as the liquid level of the overhead and underground tank respectively in the simulation; the sketch was compiled into a hex file and was uploaded to the proteus platform. The potentiometers were varied and the corresponding output was tabulated.

It can observe from Table 1 that as the potentiometer value increases, the corresponding sensor value also increases proportionally.

\subsection{Prototype}

The system was prototyped using a pair of 5 litres graduated plastic containers serving as the overhead and underground tank (separated at a height 2 meters from the overhead tank). Two ultrasonic sensors and a water pump (30 watts) were connected to the microcontroller unit. The volume of the liquid in the containers was then varied and the corresponding depth and the sensor reading were recorded. The two potentiometers in the simulation were replaced by the two Ultrasonic sensors in prototyping.

\subsection{Linearity}

Figure 5 shows a plot of the variation of the two sensor values with respect to the volume of the liquid in the container. From the plots, two linear equations were deduced. The equation for the overhead tank is shown in equation 7 and that of the underground tank is represented by equation 8 , where $y 1$ and $y 2$ are the instantaneous volume of the liquid corresponding to the value of the sensor $\mathrm{x} 1$ and $\mathrm{x} 2$ respectively with a strong correlation observed between the two sensors used.

$$
\begin{aligned}
& y_{1}=-238.6 x_{1}+996.6 \\
& y_{2}=-240.5 x_{1}+977
\end{aligned}
$$

The time of flight of the ultrasonic wave with respect to the distance of the liquid level is shown in Table 2. Equation (5) is used in conjunction with Table 2 to compute the actual height of the liquid with respect to the zero level with $\mathrm{H}=17.5 \mathrm{~cm}$.

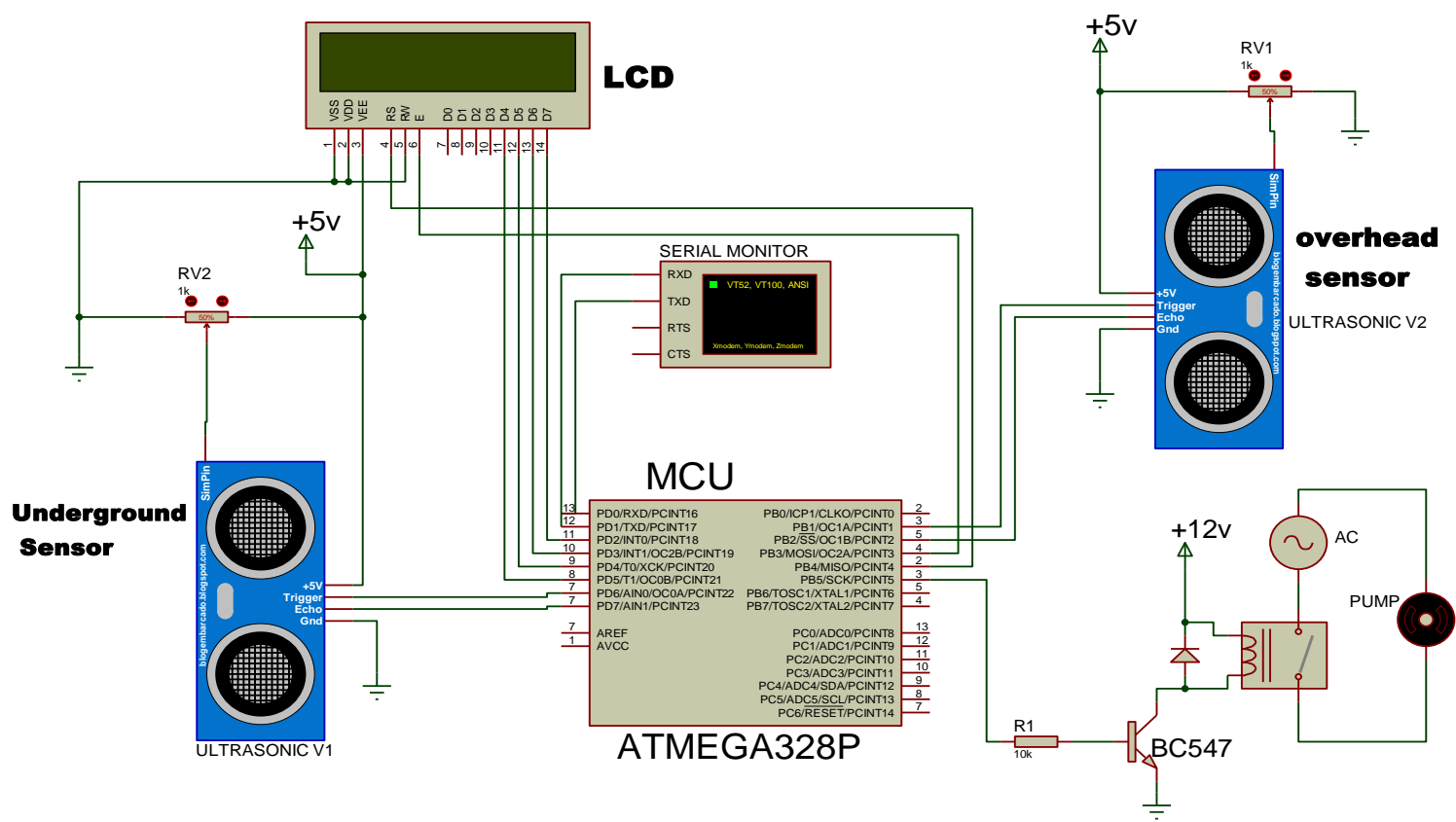

Figure 4: Simulation Diagram 
Table 1 Simulation Result for HC-SR04 Ultrasonic Range Finder

\begin{tabular}{|c|c|c|c|c|c|c|c|c|c|c|c|c|c|}
\hline SENSOR VALUE $(\mu \mathrm{s})$ & 258 & 3224 & 6449 & 9660 & 12945 & 16414 & 19404 & 22649 & 25893 & 29389 & 32377 & 35680 & 38932 \\
\hline POT. VAULUE $(\Omega)$ & 0 & 500 & 1000 & 1500 & 2000 & 2500 & 3000 & 3500 & 4000 & 4500 & 5000 & 5500 & 6000 \\
\hline
\end{tabular}

Table 2 Pulse TOF with respect to water level

\begin{tabular}{ccccccccc}
\hline \hline$d x(\mathrm{~cm})$ & 15.1 & 13.3 & 11.1 & 9.1 & 7.1 & 5.5 & 3.5 & 2.3 \\
\hline \hline$t \times 10^{-4}(\mathrm{~s})$ & 8.88 & 7.82 & 6.53 & 5.35 & 4.18 & 3.24 & 2.06 & 1.35 \\
\hline \hline
\end{tabular}

Figure 6 shows a plot of the liquid level $d l$ against the corresponding volume of the liquid $V$, this curve represents the systems stability with the change in volume of the fluid. The value of the constant of proportionality $\mathrm{K}$ in eq. 6 is deduced from the plot to be approximately $300 \mathrm{~cm}^{2}$ and that signifies the rate at which the volume increases with respect to the height of the container.
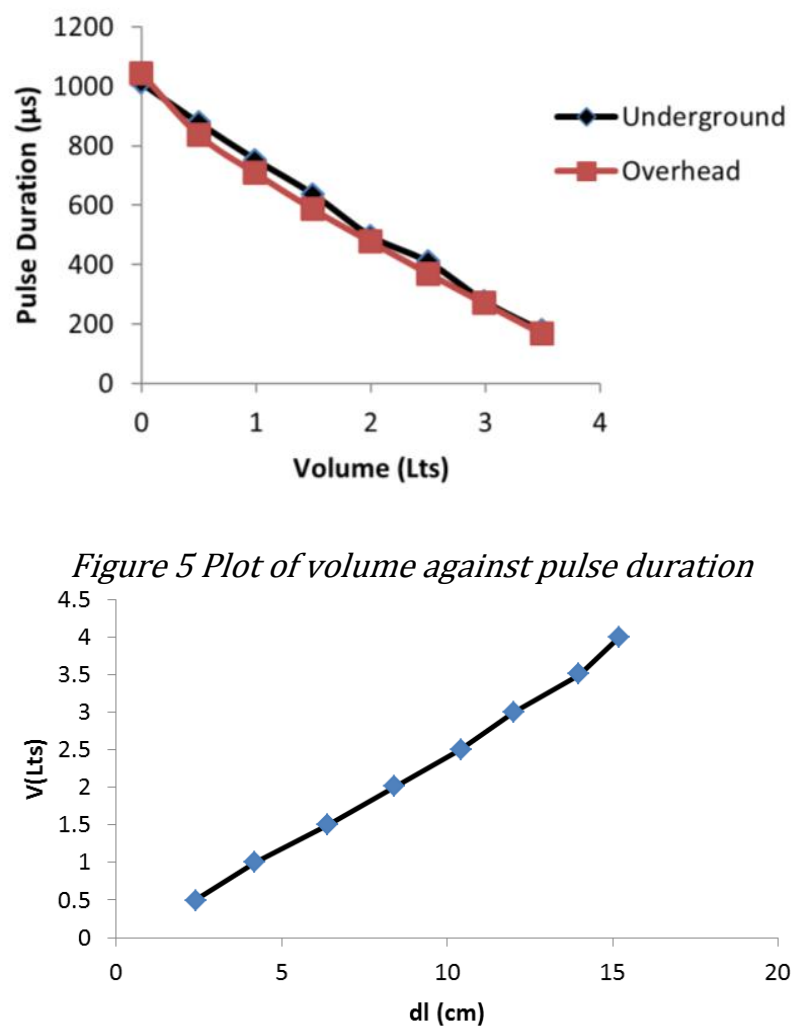

Figure 6: Plot of the Distance dl against the Volume V.

The system was designed to actuate a pumping device to pump water from the underground to the overhead tank if and only if the water in the underground reservoir is above a predefined value and the overhead tank level is below a predefined level, otherwise, the pump remains off. This is to avoid water overflow or keeping the pump $\mathrm{ON}$ while there is no water in the underground tank which may lead to pump overheating. Equations (7) and (8) are the linear equations used for the microcontroller program while equation 6 presents an alternative to determining the depth corresponding to the volume of the water with minimum error. The corresponding volume of both the reservoirs was displayed on the LCD.

\section{CONCLUSION}

In this work, a contactless non-intrusive ultrasonic based liquid level monitoring and control system was presented. The system was design based on the reflective property of ultrasonic wave by the surface of the measured liquid while the sensor intercepts the reflected wave to compute the distance of the liquid level from the sensor based on the time of flight of the wave pulse. A microcontroller was programmed to use the data from the sensor to concurrently monitor the water level of both an overhead and underground reservoir tanks. It can be seen from the results obtained that the system was successfully implemented with an optimal efficiency and a strong correlation between the simulated result and the implemented system. The system is designed to minimized water loss due to reservoir overflow, minimizes the burden of manually operated pumping systems and can also serve as a replacement to the intrusive systems that exist in the literature. The applications of the proposed system can be extended to monitor the volumes and level of other valuable fluids such as diesel, kerosene as well as hazardous chemical where human interventions may be treacherous.

\section{REFERENCES}

[1] F. Yu, B. Hong, W. Lin, C. Chao, K. Jwo, T. K. Road, T. District, N. Taipei, I. Engineering, T. K. Road, T. District, and N. T. City, "Non-Invasive Liquid Level Detection with Dielectric Capacitive Method," in 2013 IEEE 17th International Symposium on Consumer Electronics, 2013, pp. 117-118.

[2] K. Zeng and D. Zhang, "Recent progress in alkaline water electrolysis for hydrogen production and applications," Prog. Energy Combust. Sci., vol. 36, 
no. 3, pp. 307-326, 2010.

[3] G. Wranglén, "Review article on the influence of sulphide inclusions on the corrodibility of $\mathrm{Fe}$ and steel," Corros. Sci., vol. 9, no. 8, pp. 585-602, Jan. 1969.

[4] J. E. Okhaifoh, C. K. Igbinoba, and K. O. Eriaganoma, "Microcontroller Based Automatic Control for Water Pumping Machine with Water Level Indicators Using Ultrasonic Sensor," Nigerian Journal Technology, vol. 35, no. 3, pp. 579-583, 2016.

[5] E. V. Ebere and O. O. Francisca, "Microcontroller based Automatic Water level Control System," Int. J. Innov. Res. Comput. Commun. Eng., pp. 13901396, 2013.

[6] A. Abdullah, G. Anwar, T. Rahman, and S. Aznabi, "Water Level Indicator with Alarms Using PIC Microcontroller," Am. J. Eng. Res., vol. 4, no. 7, pp. 88-92, 2015.

[7] V. E. Sakharov, S. A. Kuznetsov, B. D. Zaitsev, I. E. Kuznetsova, and S. G. Joshi, "Liquid level sensor using ultrasonic Lamb waves q," Ultrasonics, vol. 41, pp. 319-322, 2003.
[8] A. Guillet, A. Vena, E. Perret, and S. Tedjini, "Design of a Chipless RFID Sensor for Water Level Detection," IEEE 15 International Symposium on Antenna Technology and Applied Electromagnetics, pp. 1-4, 2012.

[9] N. K. Kaphungkui, "Design of Low Cost and Efficient Water Level Controller," Int. J. Adv. Res. Electron. Commun. Eng., vol. 3, no. 6, pp. 671-674, 2014.

[10] C. Yang, S. Chen, and G. Yang, "Fiber optical liquid level sensor under cryogenic environment," Sensors and Actuators, vol. 94, pp. 69-75, 2001.

[11] M. Constantine, C. Anyanwu, and C. A. Edward, "Design and Implementation of a Water Level Controller," Nigerian Journal Technology, vol. 31, no. 1, pp. 1-5, 2015.

[12] L. Y. Sing and P. J. Ker, "A Microcontroller-based System for Liquid Level Detection Using Infrared Sensing," 2015 IEEE Student Conf. Res. Dev., pp. 294-299, 2015.

[13] B. Michel, Fundamentals of Acoustics, Series. Wilshire: Antony Rowe Ltd, 2006.

[14] "Reflection (physics)," Wikipedia, 2017. [Online]. Available: https//en.m.wikipedia.org/wiki/Reflection (physics). [Accessed: 01-Aug-2017]. 